ఠ

\title{
miR-486-5p attenuates tumor growth and lymphangiogenesis by targeting neuropilin- 2 in colorectal carcinoma
}

This article was published in the following Dove Press journal:

OncoTargets and Therapy

19 May 2016

Number of times this article has been viewed

\section{Chengxia Liu \\ Ming Li \\ Yingbin $\mathrm{Hu}$ \\ Ning Shi \\ Haisheng Yu \\ Haiyan Liu \\ Haifeng Lian}

Department of Gastroenterology, the Affiliated Hospital of Binzhou

Medical University, Binzhou, Shandong

Province, People's Republic of China
Correspondence: Haifeng Lian

Department of Gastroenterology, the Affiliated Hospital of Binzhou Medical University, 66I Huanghe 2nd Road, Binzhou, Shandong, 256603, People's Republic of China

Tel +86 I5I 66098756

Email lianhaifeng0I@I63.com

\begin{abstract}
Increasing evidence suggests that microRNAs are associated with many important biologic processes in carcinogenesis. Despite ample research revealing the dysregualtion of miR-486-5p in various cancers, little is known about the roles of miR-486-5p in colorectal carcinoma (CRC). In this study, we investigated the biological functions and molecular mechanisms of miR-486-5p in CRC growth and invasion, discussing the potential of using miR-486-5p as a biomarker for colorectal cancers. Our data revealed that miR-486-5p was significantly downregulated in CRC tissues compared with the paracancer tissue by quantitative real-time polymerase chain reaction and that miR-486-5p was downregulated to a greater extent in advanced stage cancer (stage III and IV) as compared to early stage cancer (stage I and II). Luciferase reporter assay verified that neuropilin-2 was a direct functional target of miR-486-5p in the CRC cells, and upregulation of miR-486-5p in CRC cells negatively correlated with the expression of neuropilin-2. Furthermore, overexpression of miR-486-5p inhibited the tumor growth and lymphangiogenesis in nude mice, which was reversed by overexpression of neuropilin-2. Taken together, our study suggested miR-486-5p might be a suppressor of CRC.
\end{abstract}

Keywords: miR-486-5p, colorectal carcinoma, neuropilin-2, tumor growth, lymphangiogenesis

\section{Introduction}

Colorectal cancer is one of the most common cancers and the fourth most frequent cause of cancer-related deaths worldwide. ${ }^{1,2}$ Despite the recent advances in diagnosis and treatment, the prognosis for colorectal carcinoma (CRC) patients is still poor. Colorectal cancer has a high mortality rate, with relapse and metastasis as the inevitable outcomes in most patients. ${ }^{3,4}$ Metastasis of CRC via lymphatics usually indicates a poor prognosis, ${ }^{5,6}$ and lymphangiogenesis critically contributes to the lymphatic metastasis of CRC; however, the underlying mechanism of CRC lymphangiogenesis remains largely elusive. ${ }^{7}$

MicroRNAs (miRNAs) have been identified as a class of short noncoding RNA sequences that negatively regulate their target genes at the posttranscriptional level through RNA interference ${ }^{8}$ and modulate many cellular processes, including proliferation, apoptosis, metastasis, and chemoresistance. ${ }^{9,10}$ Evidence has indicated that miRNA-486-5P functions as a cancer suppressor in several carcinogenesis; for example, Wang et $\mathrm{al}^{11}$ reported that reduced expression of miR-486-5p was inversely associated with advanced stage and lymph node metastasis of non-small-cell lung cancer, and $\mathrm{Oh}$ et $\mathrm{al}^{12}$ reported that miR-486-5p was significantly downregulated in the primary gastric cancers and gastric cancer cell lines. Conversely, miR-486-5p 
is expressed in Down Syndrome myeloid leukemias and enhances their aberrant erythroid phenotype. ${ }^{13}$ However, its role in CRC progression is unknown.

Neuropilin-2 (NRP2) is one of the most important regulators of lymphangiogenesis. NRP2 is a non-tyrosine kinase transmembrane glycoprotein, and its receptor has been identified on endothelial cells and tumor cells. ${ }^{14,15}$ It is predominantly expressed in venous and lymphatic endothelial cells, as well as cells in neural crest origin tumors, such as melanoma, glioblastoma, and neuroblastoma. ${ }^{16}$ NRP2 has been shown to play an inhibitory role in tumor growth, migration, and invasion of pancreatic adenocarcinoma cancer. ${ }^{17,18}$ NRP2 is critical for tumor angiogenesis and lymphangiogenesis, ${ }^{19}$ and downregulation of NRP2 reduced tumor growth and metastasis in osteosarcoma. ${ }^{20}$ In fact, NRP2 knockout mice are deficient in small lymphatic vessels and lymphatic capillaries. ${ }^{21}$ Recently, NRP2 was reported to play a critical role in lymph node metastasis as a regulator of lymphangiogenesis through an indirect pathway involving vascular endothelial growth factor- $\mathrm{C}$ and can also regulate vascular endothelial growth factor-D-induced invasion and migration. ${ }^{22}$ However, to date, the function of NRP2 in colorectal cancer and the mechanism underlying its regulation remain poorly understood.

In this study, we hypothesized that miR-486-5p regulates NRP2 expression in CRC. We found that targeting NRP2 or miR-486-5p significantly inhibits tumor growth and lymphangiogenesis in nude mice. Thus, our data suggest that miR-486-5p may function as a tumor suppressor to influence the tumor growth and lymphangiogenesis of CRC.

\section{Materials and methods Specimens}

A total of 66 fresh CRCs specimens and peritumoral normal colorectal tissues were obtained from the affiliated Hospital of Binzhou Medical University (Binzhou, People's Republic of China). All the samples were diagnosed as CRC without chemotherapy or radiotherapy before surgery, and subgrouped into stage I and II group, stage III group, and stage IV group according to the TNM classification criteria. All specimens were frozen in liquid nitrogen for further study. Personally signed consent forms were obtained from each patient, and the patient's personal information was protected. This study was approved by the Research Ethics Committee of Binzhou Medical University.

\section{Cell culture}

Human CRC cell lines SW620 and HT-29 were purchased from Boster Company (Boster, Wuhan, People's Republic of China). Human CRC cells were cultured in Roswell Park
Memorial Institute (RPMI-1640) (Hyclone, Waltham, MA, USA), with $10 \%$ fetal bovine serum (Hyclone). Cells were maintained in humidified incubators $\left(5 \% \mathrm{CO}_{2}\right)$ at $37^{\circ} \mathrm{C}$. The protocol of using the human CRC cell line SW620 and HT-29 was approved by the Research Ethics Committee of Binzhou Medical University.

\section{Luciferase reporter assay}

NRP2 3'UTR was cloned into the multiple cloning sites of pMIR-GLO Vector (GenePharma, Shanghai, People's Republic of China) between the SacI and HindIII restriction sites. The SW620 cells were cotransfected with $2 \mu \mathrm{g}$ of the 3'UTR-luciferase reporter vector and $2 \mu \mathrm{g}$ of miR-486-5p or negative controls using Lipofectamine 2000 reagent (Thermo Fisher Scientific, Waltham, MA, USA) in 12-well plates. An amount of $2 \mu \mathrm{g}$ of pMIR-GLO Vector plasmid was added into the well at the same time as a normalizing control. Cells were harvested after 72 hours incubation. Luciferase values were determined using the Dual-Luciferase Reporter Assay System (Beyotime Biotechnology, Beijing, People's Republic of China).

\section{Cell transfection}

All cells were transfected with $500 \mathrm{ng} / \mu \mathrm{L}$ miR486-5P mimics or the negative control (GenePharma) using Lipofectamine 2000 (Invitrogen), which were prepared in RPMI-1640 Reduced Serum Medium after plating for 12 hours. Then, microscopy, quantitative real-time polymerase chain reaction (qRT-PCR), and Western blot were used to verify transfection efficiency.

\section{Quantitative real-time PCR}

Total RNA from cell lines and the fresh tissue samples were extracted using TRizol reagent (Invitrogen). Only those RNA samples with 260/280 ratios of 1.8-2.0 were used for further investigation. The isolated RNA was reverse transcribed and amplified using the TaqMan MicroRNA Assays (Takara Bio Inc, Kusatsu, Shiga, Japan) according to the manufacturer's protocol. qRT-PCR was performed by using an Applied RT-3000 real-time PCR system. Primers for miR-486-5p and U6 internal control were synthesized by RiboBio Company (RiboBio, Guangzhou, People's Republic of China). The mRNA expression levels were determined using the $2^{-\Delta \Delta \mathrm{Ct}}$ method.

\section{Western blotting}

Proteins from cells and tumor tissues were extracted in radioimmunoprecipitation assay buffer; after centrifugation at 12,000 rpm for 10 minutes at $4^{\circ} \mathrm{C}$, proteins in the supernatants were separated by $6 \%$ sodium dodecyl sulfate polyacrylamide gel 
electrophoresis and transferred to polyvinylidene fluoride membrane (Beyotime Biotechnology). After blocking with 7\% nonfat milk in Tris Buffered Saline with Tween ${ }^{\circledR} 20$, membranes were immunoblotted with antibodies as indicated, followed by horseradish peroxidase -conjugated-linked secondary antibodies (Santa Cruz Biotechnology, Dallas, TX, USA). NRP2 antibodies were purchased from Abcam (Abcam, Cambridge, UK). Protein levels were normalized to total glyceraldehyde 3-phosphate dehydrogenase (Santa Cruz Biotechnology).

\section{Tumorigenicity assay in nude mice}

For this experiment, $1 \times 10^{7} \mathrm{CRC}$ cells were suspended in 200 $\mu \mathrm{L}$ RPMI-1640 and then injected subcutaneously into the dorsal sites of 6 week-old male nude mice. Tumor formation was monitored $\sim 1$ week. Some mice were peritumoral injected with miR486-5p mimics every 3 days for 2 weeks. Phosphatebuffered saline (PBS) or mock injections were performed on some animals as negative controls. The tumor volume $\left(\mathrm{mm}^{3}\right)$ was measured every 3 days and calculated using the following formula: volume $=0.5 \times \mathrm{L} \times \mathrm{W}^{2}$ (in millimeters), where $\mathrm{L}$ represented the length of the tumor and $\mathrm{W}$ represented the width of the tumor. The mice were sacrificed and the tumors were collected after 3 weeks; the tumors were bisected with one half being fixed in 4\% formalin and embedded in formaldehyde and the remainder snap-frozen in liquid nitrogen for histological studies. The animal experiments were approved by the Animal Care and Use Committee of Binzhou Medical University. All protocols were performed in accordance with the ethical principles and guidelines for scientific experiments on animals of the Swiss Academy of Medical Sciences (1995).

\section{Immunohistochemistry}

Tumor samples were fixed for 24 hours at $4{ }^{\circ} \mathrm{C}$ in $4 \%$ formaldehyde, dehydrated and embedded in paraffin, and sectioned (thickness, $4 \mu \mathrm{m}$ ) for immunohistochemical analysis of NRP2 and lymphatic microvessel density. LYVE-1 (Abcam) was used to monitor the numbers of lymphatic microvessels in the tumors. Briefly, slides were washed and incubated with $3 \%$ $\mathrm{H}_{2} \mathrm{O}_{2}$ for 20 minutes to quench endogenous peroxide activities and then were blocked with $1 \%$ bovine serum albumin in PBS for 40 minutes at $37^{\circ} \mathrm{C}$. Monoclonal antibodies against NRP2 (1:200) and LYVE-1 (1:100) were used as the primary antibody for detecting protein expression. Immunodetection was performed by incubation with a specific biotinylated secondary antibody followed by use of the kit (ZSGB-BIO, Beijing, People's Republic of China). Slides were examined under a light microscope (Olympus Corporation, Tokyo, Japan) with a $\times 40$ magnification.

\section{Statistical analysis}

All computations were carried out using the Statistical Package of SPSS version 13.0 for Windows (SPSS Inc., Chicago, IL, USA). The statistical analysis was performed by one-way analysis of variance (when $>3$ groups). Differences were considered statistically significant when $P<0.05$.

\section{Results}

miR-486-5p is downregulated in CRC and inversely correlated with lymphangiogenesis

To explore the functional role of miR-486-5p in CRC, we first compared the expression levels in clinical CRCs and paired adjacent normal tissues from 66 cases of CRC patients. By stem-loop qRT-PCR, miR-486-5p expression was reduced in CRC specimens compared with adjacent normal tissues and miR-486-5p level was correlated with lymphangiogenesis (Figure 1A). Furthermore, we divided all the 66 colonrectal cancer (CTC) patients into three groups according to the TNM classification, stage I and II group, stage III group and stage IV group, and found that the miR-486-5p level was downregulated to a greater extent in advanced stage cancer (stage III and IV) as compared to early stage cancer (stage I and II) (Figure 1B). This result indicated that reduced miR486-5p expression was a frequent event in human $\mathrm{CRC}$, and since the majority of the CTC tumors spread via lymphatic routes, this result also indicated that $\mathrm{miC}-486-5 \mathrm{p}$ may be involved in CRC lymphangiogenesis.

\section{miR-486-5p directly targets NRP2 in CRC cells}

To validate that NRP2 is indeed directly targeted by miR-486-5p, we investigated whether miR-486-5p recognizes the 3'UTR of NRP2 mRNA using a dual-luciferase reporter assay. By searching the miRNA target prediction website (www.targetscan.org), we found that NRP2 gene is a potential target of miR-486-5p. We cloned the 3'UTR sequence containing the predicted binding sites of NRP2 into the pMIR-GLO Vector. We found that transfection of vector-miR-486-5p significantly suppressed the luciferase activity of the NRP2 promoter in the SW620 and HT-29 cells (Figure 2A).

Furthermore, to validate that NRP2 is indeed directly targeted by miR-486-5p, we examined the expression levels of miR-486-5p in total RNA extracted from transfected CRC cells and paired CRC cells. We found that transfection of miR-486-5p into SW620 and HT-29 CTC cells 

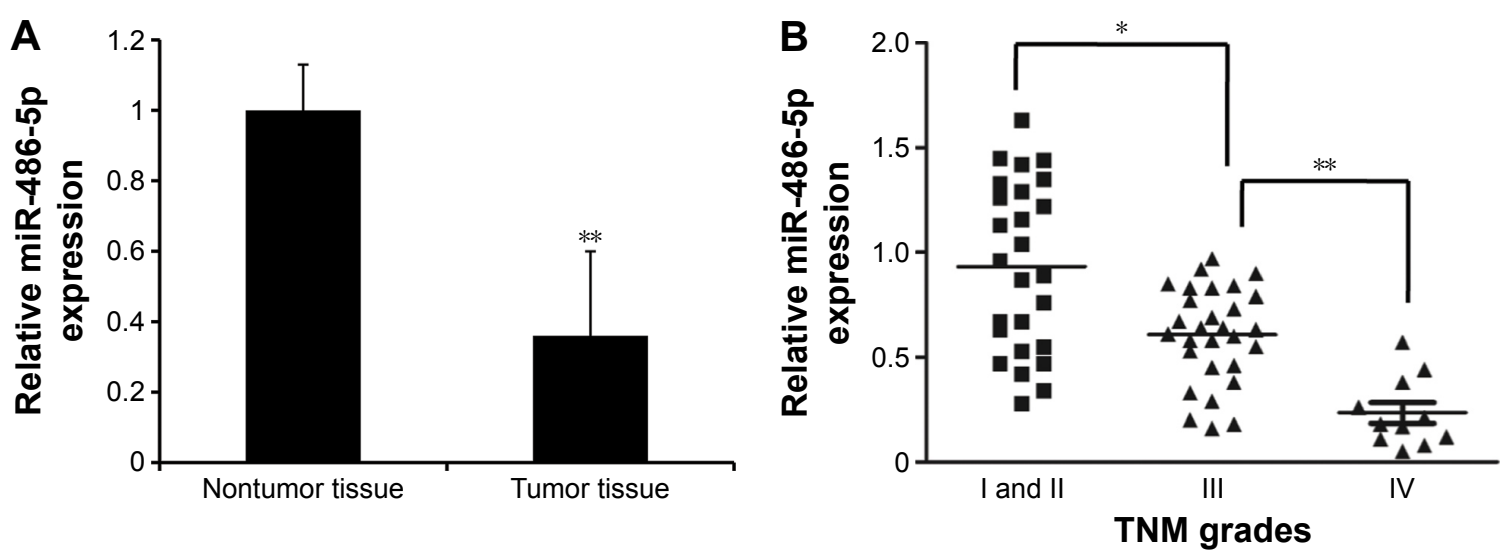

Figure I miR-486-5p expression in CRC.

Notes: (A) Relative expression of miR-486-5p in 66 CRC tissues compared with their matched adjacent nontumor tissues. (B) miR-486-5p expression in three CTC tumor subgroups according to the TNM classification: stage I and II group containing 26 patients, stage III group containing 29 patients, and stage IV containing eleven patients. miR486-5p was quantified by qRT-PCR with specific primers for miR-486-5p and U6 snRNA. Square represents patients of TNM grade I and II, and triangle represents patients of TNM grade III and IV. $* P<0.05 ; * * P<0.01$.

Abbreviations: CRC, colorectal carcinoma; qRT-PCR, quantitative real-time polymerase chain reaction; TNM, TNM Classification of Malignant Tumours; CTC, colonrectal cancer.

indeed increased the miR-486-5p level as detected by qPCR (Figure 2B), and compared with the SW620 cells and HT-29 cells transfected with nontarget control, the expression of NRP2 in these cells transfected with miR-486-5p expressing vectors was significantly decreased both at mRNA and protein levels (Figure 2C and D).

A

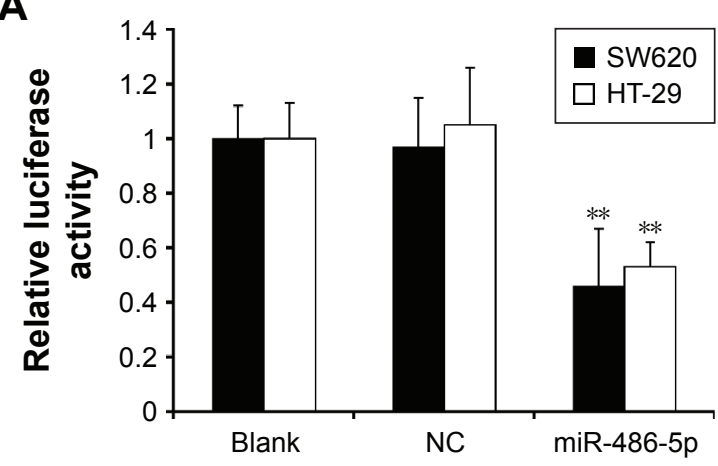

C

SW620

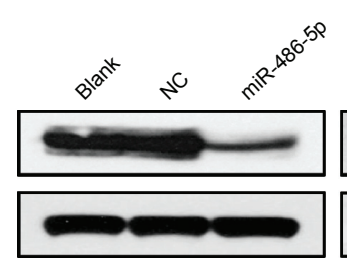

HT-29

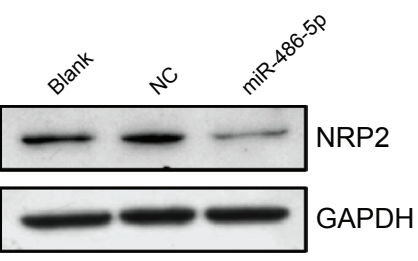

Overexpression of miR-486-5p inhibited tumor growth and lymphangiogenesis in vivo

To further confirm the growth-attenuating effect of miR486-5p on CRC, a xenograft tumor growth assay was performed. Nude mice were injected with either SW620 cells

B

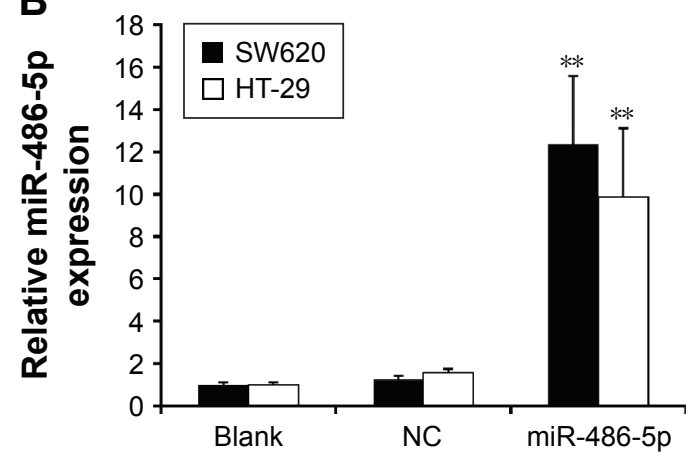

D

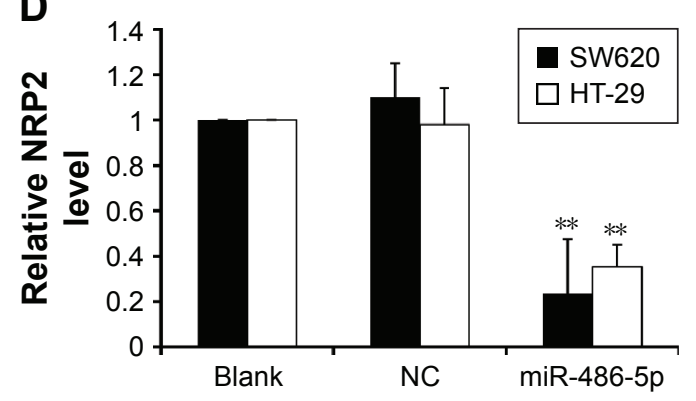

Figure 2 NRP2 is a target of miR-486-5p.

Notes: (A) Effect of miR-486-5p on NRP2 expression as determined by a luciferase reporter assay. The data were normalized by determining the firefly and Renilla luciferase activities measured at 72 hours posttransfection; SW620 and HT-29 cells were transfected with miR-486-5p mimic (miR-486-5p group), mimic control (NC group), and blank control (blank group). (B) Transfection of miR-486-5p mimics to SW620 and HT-29 cells increases the expression of miR-486-5p detected by qRT-PCR. (C) NRP2 protein expression level was significantly decreased in miR-486-5p-transfected SW620 and HT-29 cells. (D) Relative NRP2 protein levels in figure (C) were quantified using the gray scale quantification analysis. $* * P<0.01$.

Abbreviations: GAPDH, glyceraldehyde 3-phosphate dehydrogenase; NRP2, neuropilin-2; qRT-PCR, quantitative real-time polymerase chain reaction; NC, nontarget control. 
overexpressing miR-486-5p or SW620 cells transfected with the empty vector and the subcutaneous tumor volume growth curve miR-486-5p was determined in vivo. The tumor volume was significantly lower in the miR-486-5p overexpressing mice as compared to the control mice during 3 weeks of culture (Figure 3A). After 3 weeks, the tumors were harvested. The total weight of the xenograft tumors was significantly lower in miR-486-5p mice when compared with the control mice (Figure 3B). Correspondingly, the expression of NRP2 was significantly decreased in miR-486-5p transfected tumors, compared with the control group (Figure 3C and D).

Furthermore, NRP2 was positively associated with the lymphatic vessel density of the xenografts (Figure 4A and B). LYVE-1 was used to monitor the numbers of lymphatic microvessels in the peritumoral regions, which were evaluated in five independent fields for each of the three tumors per group (Figure 4C). Thus, these results revealed that miR-486-5p expression could attenuate the proliferation and lymphangiogenesis of CRC cells in vivo.

\section{Discussion}

Colorectal cancer is a malignant gastrointestinal tumor with high prevalence in People's Republic of China. The development and progression of CRC are caused by dysregulation of multiple genes that are essential for cell biological processes. However, the carcinogenesis underlying molecular mechanism remains largely unclear. miRNAs play important roles in a variety of cancers in which their expression patterns are altered and functioned as either oncogenes or tumor suppressors. ${ }^{23}$ While we have made great strides in understanding the role of miRNAs in tumorigenesis, there are many unanswered questions regarding the involvement of miRNAs in progression and metastasis of CRC. In particular, the function of miR-486-5p and its regulated targets in CRC remains unknown. This study is the first to show that the expression of miR-486-5p is significantly downregulated in CRC samples compared with that in the adjacent normal tissues, and expression level of miR-486-5p is negatively correlated with CTC tumor grade. The low expression of miR-486-5p in colorectal cancers suggests that it might have anticancer effects.

Previous studies have reported that miR-486-5p affected the occurrence and development of carcinoma. ${ }^{24}$ For instance, overexpression of miR-486-5p remarkably suppressed HCC cell proliferation, migration, and invasion in vitro, and inhibited HCC growth in vivo by targeting PIK3R1 and AKT. ${ }^{25}$ Furthermore, miR-486-5p was downregulated in breast cancer and significantly inhibited
A

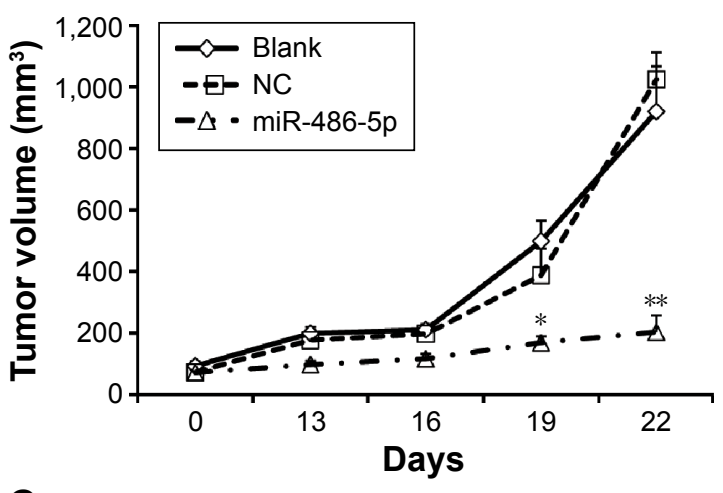

C

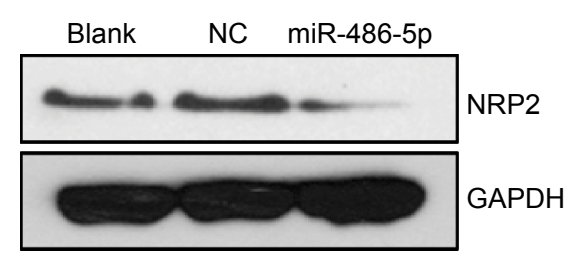

B
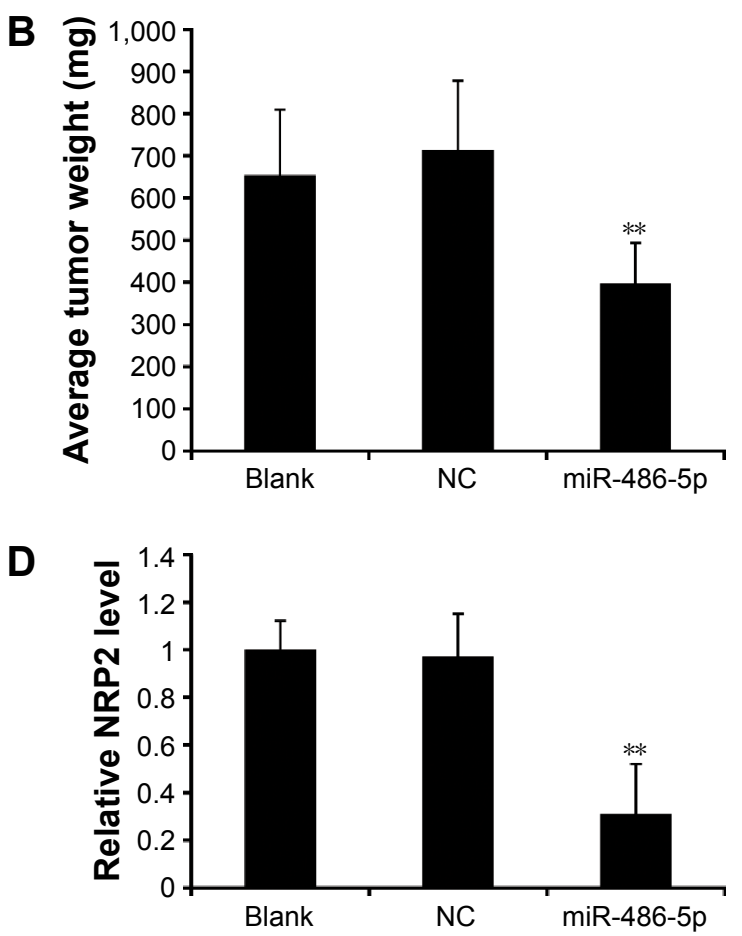

Figure 3 miR-486-5p inhibited xenograft tumor growth by targeting NRP2 in vivo.

Notes: (A) Groups of nude mice were implanted subcutaneously with SW620/PBS cells (Blank group), SW620/mimic-control cells (NC group), or SW620/miR-486-5p cells (miR-486-5p group), Tumor volumes were recorded at the indicated times. (B) The tumor mass was determined when the mice were sacrificed. (C) NRP2 expression in tumors was examined by Western blot. (D) Quantitative analysis of the NRP2 protein level using a gray scan analysis. One representative tumor mass group from three independent experiments with similar results is shown. $* P<0.05, * * P<0.01$.

Abbreviations: GAPDH, glyceraldehyde 3-phosphate dehydrogenase; NRP2, neuropilin-2; PBS, phosphate-buffered saline; NC, nontarget control. 

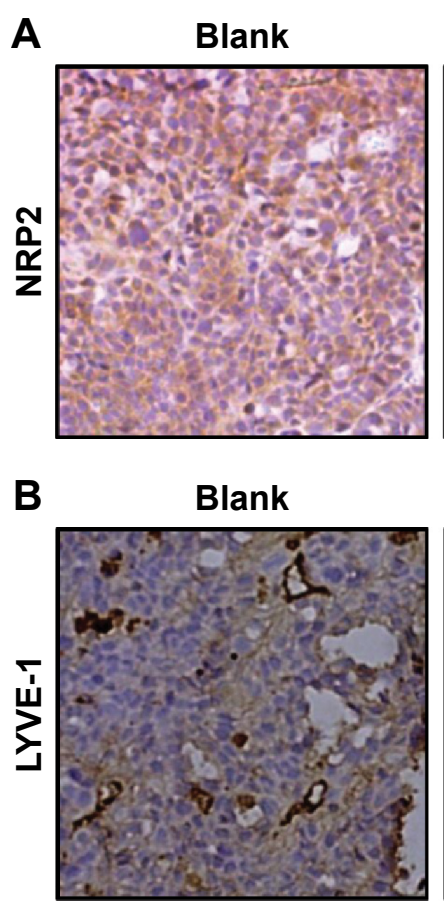

\section{c}

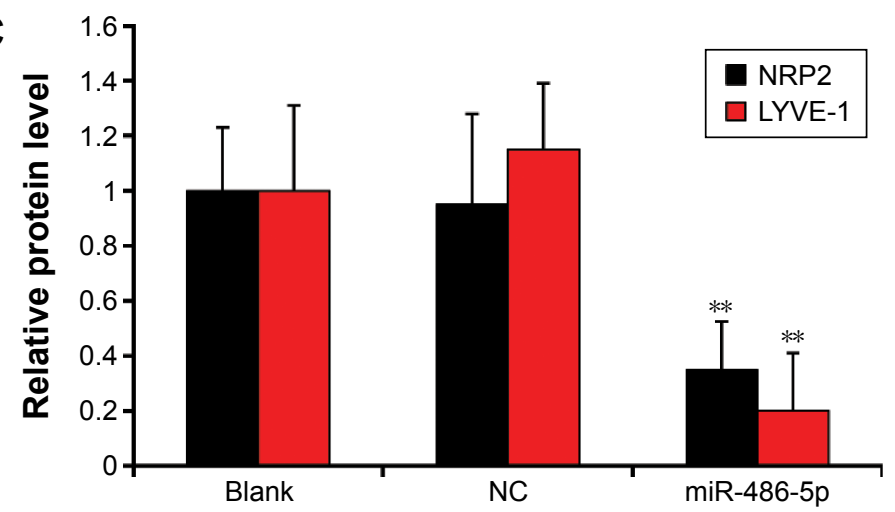

Figure 4 NRP2 and LYVE-I expression in nude mouse xenografts.

Notes: (A) Western blot analysis of NRP2 protein expression in three independent experimental groups. (B) Immunohistochemical staining for NRP2 and LYVE-I from nude mouse xenograft tumors. Original magnification, $\times 40$. (C) Quantification of NRP2 and LYVE-I in each group. $* * P<0.0$ I.

Abbreviations: NC, nontarget control; NRP2, neuropilin-2.

the proliferation, apoptosis, and invasion in vitro and in vivo by regulating PIM-1. ${ }^{26}$ Accordingly, our study revealed that overexpression of miR-486-5p could suppress the proliferation, tumor growth, and lymphangiogenesis of CRC in vitro and in vivo.

We identified NRP2 as a direct target of miR-486-5p by bioinformatics prediction (www.targetscan.org). We observed that NRP2 levels were significantly decreased in the miRNA-486-5p overexpression of CRC cells. Most importantly, as expected, the density of lymphatic microvessels and tumor mass of xenografts derived from miR-486-5p overexpressing SW620 cells were significantly lower than those from the xenografts derived from control SW620 cells. Our findings, for the first time, revealed that
miR-486-5p mediates CRC angiogenesis and lymphangiogenesis via targeting NRP2.

In summary, the present study indicated that miR486-5p expression was decreased in CRC tissues. We have identified that miR-486-5p attenuated the proliferation and lymphangiogenesis of CRC cells in vivo by directly targeting NRP2. MiR-486-5p may provide a potential therapeutic target of metastatic CRCs in the future. We hope that our investigation can facilitate further exploration of the molecular mechanisms of miR-486-5p in CRC.

\section{Acknowledgments}

This study was supported by the Natural Science Foundation of Shandong Province (No ZR2014HQ072; ZR2015LH083); 
the Science and Technology Development Plan of Medicine and Health Care of Shandong Province (No 2014WS0490); and the Science and Technology Development Plans of Binzhou (No 2014ZC0116).

\section{Disclosure}

The authors report no conflicts of interest in this work.

\section{References}

1. Wang F, Wong SC, Chan LW, Cho WC, Yip SP, Yung BY. Multiple regression analysis of mRNA-miRNA associations in colorectal cancer pathway. Biomed Res Int. 2014;2014:676724.

2. Ferlay J, Soerjomataram I, Dikshit R, et al. Cancer incidence and mortality worldwide: sources, methods and major patterns in GLOBOCAN 2012. Int J Cancer. 2015;136(5):E359-E386.

3. Markowitz SD, Dawson DM, Willis J, Willson JK. Focus on colon cancer. Cancer Cell. 2002;1(3):233-236.

4. Labianca R, Nordlinger B, Beretta GD, Brouquet A, Cervantes A; ESMO Guidelines Working Group. Primary colon cancer: ESMO Clinical Practice Guidelines for diagnosis, adjuvant treatment and follow-up. Ann Oncol. 2010;21 Suppl 5:v70-v77.

5. Stacker SA, Achen MG, Jussila L, Baldwin ME, Alitalo K. Lymphangiogenesis and cancer metastasis. Nat Rev Cancer. 2002;2(8):573-583.

6. Stacker SA, Baldwin ME, Achen MG. The role of tumor lymphangiogenesis in metastatic spread. FASEB J. 2002;16(9):922-934.

7. Ou JJ, Wei X, Peng Y, et al. Neuropilin-2 mediates lymphangiogenesis of colorectal carcinoma via a VEGFC/VEGFR3 independent signaling. Cancer Lett. 2015;358(2):200-209.

8. Chen K, Rajewsky N. The evolution of gene regulation by transcription factors and microRNAs. Nat Rev Genet. 2007;8(2):93-103.

9. Osaki M, Takeshita F, Sugimoto Y, et al. MicroRNA-143 regulates human osteosarcoma metastasis by regulating matrix metalloprotease-13 expression. Mol Ther. 2011;19(6):1123-1130.

10. Lv L, Deng H, Li Y, et al. The DNA methylation-regulated miR-193a-3p dictates the multi-chemoresistance of bladder cancer via repression of SRSF2/PLAU/HIC2 expression. Cell Death Dis. 2014;5:e1402.

11. Wang J, Tian X, Han R, et al. Downregulation of miR-486-5p contributes to tumor progression and metastasis by targeting protumorigenic ARHGAP5 in lung cancer. Oncogene. 2014;33(9):1181-1189.

12. Oh HK, Tan AL, Das K, et al. Genomic loss of miR-486 regulates tumor progression and the OLFM4 antiapoptotic factor in gastric cancer. Clin Cancer Res. 2011;17(9):2657-2667.
13. Shaham L, Vendramini E, Ge Y, et al. MicroRNA-486-5p is an erythroid oncomiR of the myeloid leukemias of Down syndrome. Blood. 2015;125(8):1292-1301.

14. Staton CA, Kumar I, Reed MW, Brown NJ. Neuropilins in physiological and pathological angiogenesis. $J$ Pathol. 2007;212(3):237-248.

15. Dallas NA, Fan F, Gray MJ, et al. Functional significance of vascular endothelial growth factor receptors on gastrointestinal cancer cells. Cancer Metastasis Rev. 2007;26(3-4):433-441.

16. Bielenberg DR, Pettaway CA, Takashima S, Klagsbrun M. Neuropilins in neoplasms: expression, regulation, and function. Exp Cell Res. 2006; 312(5):584-593.

17. Timoshenko AV, Rastogi S, Lala PK. Migration-promoting role of VEGF-C and VEGF-C binding receptors in human breast cancer cells. Br J Cancer. 2007;97(8):1090-1098.

18. Dallas NA, Gray MJ, Xia L, et al. Neuropilin-2-mediated tumor growth and angiogenesis in pancreatic adenocarcinoma. Clin Cancer Res. 2008; 14(24):8052-8060.

19. Caunt M, Mak J, Liang WC, et al. Blocking neuropilin-2 function inhibits tumor cell metastasis. Cancer Cell. 2008;13(4):331-342.

20. Ji T, Guo Y, Kim K, et al. Neuropilin-2 expression is inhibited by secreted Wnt antagonists and its down-regulation is associated with reduced tumor growth and metastasis in osteosarcoma. Mol Cancer. 2015; 14:86.

21. Yuan L, Moyon D, Pardanaud L, et al. Abnormal lymphatic vessel development in neuropilin 2 mutant mice. Development. 2002;129(20): 4797-4806.

22. Yasuoka H, Kodama R, Hirokawa M, et al. Neuropilin-2 expression in papillary thyroid carcinoma: correlation with VEGF-D expression, lymph node metastasis, and VEGF-D-induced aggressive cancer cell phenotype. J Clin Endocrinol Metab. 2011;96(11):E1857-E1861.

23. Tutar L, Tutar E, Tutar Y. MicroRNAs and cancer; an overview. Curr Pharm Biotechnol. 2014;15(5):430-437.

24. Yan Q, Yuan Y, Yankui L, et al. The expression and significance of CXCR5 and MMP-13 in colorectal cancer. Cell Biochem Biophys. Epub 2015 Mar 1.

25. Huang XP, Hou J, Shen XY, et al. MicroRNA-486-5p, which is downregulated in hepatocellular carcinoma, suppresses tumor growth by targeting PIK3R1. FEBS J. 2015;282(3):579-594.

26. Zhang G, Liu Z, Cui G, Wang X, Yang Z. MicroRNA-486-5p targeting PIM-1 suppresses cell proliferation in breast cancer cells. Tumour Biol. 2014;35(11):11137-11145.
OncoTargets and Therapy

\section{Publish your work in this journal}

OncoTargets and Therapy is an international, peer-reviewed, open access journal focusing on the pathological basis of all cancers, potential targets for therapy and treatment protocols employed to improve the management of cancer patients. The journal also focuses on the impact of management programs and new therapeutic agents and protocols on

\section{Dovepress}

patient perspectives such as quality of life, adherence and satisfaction. The manuscript management system is completely online and includes a very quick and fair peer-review system, which is all easy to use. Visit http://www.dovepress.com/testimonials.php to read real quotes from published authors. 